\title{
Propagators of Generalized Schrödinger Equations Related by First-order Supersymmetry
}

\author{
A. Schulze-Halberg
}

\begin{abstract}
We construct an explicit relation between propagators of generalized Schrödinger equations that are linked by a first-order supersymmetric transformation. Our findings extend and complement recent results on the conventional case [1].
\end{abstract}

Keywords: generalized Schrödinger equation, propagator, supersymmetry.

\section{Introduction}

The formalism of supersymmetry (SUSY) is a wellknown tool for identifying integrable cases of the Schrödinger equation and for generating the corresponding solutions. The principal idea is to relate two Schrödinger equations and their solutions via a supersymmetrical (or Darboux [3]) transformation, such that known solutions of one equation can be mapped onto solutions of the second equation. There is a vast amount of literature on the SUSY formalism and its applications, for details we refer the reader to $[5,2]$ and references therein. While the concept of the SUSY formalism as a method for generating solutions is popular, it is less known that two Schrödinger equations related by a SUSY transformation (SUSY partners) share more properties than the link between their solutions. In particular, SUSY establishes a connection between the propagators and the Green's functions of the underlying partner equations: the propagators are linked by means of an integral expression [1], while the Green's functions satisfy a simple trace formula $[9,6]$. It is interesting to note that this trace formula persists under generalization of the Schrödinger equation to the effective mass case [8] or to a generalized Sturm-Liouville problem [7]. Due to the close relation between Green's function and the propagator, one would expect that the propagator relation found in [1] also extends to generalized cases of the Schrödinger equation. This is in fact true, as will be shown in this note. We restrict ourselves to first-order SUSY transformations of generalized Schrödinger equations, a brief review of which and of related theory is given in section 2 . Subsequently, the explicit integral formula that links the propagators of our SUSY partner equations is done in section 3 .

\section{Preliminaries}

In the following we briefly summarize basic facts about generalized Schrödinger equations, their
SUSY formalism, propagators and Green's functions.

Generalized Schrödinger equation. We consider the following generalized Sturm-Liouville problem on the real interval $(a, b)$, equipped with Dirichlet boundary conditions:

$$
\begin{aligned}
f(x) \psi^{\prime \prime}(x)+f^{\prime}(x) \psi^{\prime}(x)+ & \\
{[E h(x)-V(x)] \psi(x) } & =0, \quad x \in(a, b) \\
\psi(a)=\psi(b) & =0 .
\end{aligned}
$$

Here $f, h, V$ are smooth, real functions, with $f, h$ positive and bounded in $a$ and $b$. The constant $E$ will be referred to as energy, and in solutions of (1), (2) that belong to the discrete spectrum, $E$ stands for the spectral value. Any solution $\psi$ of (1), (2) belonging to a value $E$ from the discrete spectrum, is located in the weighted Hilbert space $L_{h}^{2}(a, b)$ with weight function $h$ [4]. The lowest value of the discrete spectrum will be called the ground state and denoted by $E_{0}$ with corresponding solution $\psi_{0}$. The interval $(a, b)$ can be unbounded, that is, $a$ or $b$ can represent minus infinity or infinity, respectively (however, if $a$ and/or $b$ are finite, then we require $f, h, V$ to be continuous there). We see that the problem (1), (2) can be singular, which means that its spectrum can admit a continuous part. Equation (1) will be referred to as the generalized Schrödinger equation, since its special cases are frequently encountered in Quantum Mechanics, such as the Schrödinger equation for effective mass or with a linearly energy-dependent potential. In the quantum-mechanical context, $E$ denotes the energy associated with a solution $\psi$, and $V$ stands for the potential.

Generalized SUSY formalism. We summarize results from [10]. The boundary-value problem (1), (2) can be linked to another problem of the same kind by means of the SUSY transformation method. Consider

$$
f(x) \phi^{\prime \prime}(x)+f^{\prime}(x) \phi^{\prime}(x)+
$$




$$
\begin{aligned}
{[E h(x)-U(x)] \phi(x) } & =0, \quad x \in(a, b) \\
\phi(a)=\phi(b) & =0
\end{aligned}
$$

where the same settings imposed for (1), (2) apply. Clearly, a solution $\phi=\phi(x)$ and the potential $U=U(x)$ are in general different from their respective counterparts $\psi$ and $V$. Now, suppose that $\psi$ and $u$ are solutions of the boundary-value problem (1), (2) and of equation (1) at real energies $E$ and $\lambda \leq E$, respectively. Define the SUSY transformation of $\psi$ as

$$
\begin{aligned}
D_{u, x} \psi(x)= & \sqrt{\frac{f(x)}{h(x)}} \frac{W(u, \psi)(x)}{u(x)}= \\
& \sqrt{\frac{f(x)}{h(x)}}\left[-\frac{u^{\prime}(x)}{u(x)} \psi(x)+\psi^{\prime}(x)\right],
\end{aligned}
$$

where $W(u, \psi)$ stands for the Wronskian of the functions $u, \psi$ and the second index of $D$ denotes the variable which the derivatives in the Wronskian apply to. The function $\phi=D_{u, x} \psi$ as defined in (5) solves the boundary-value problem (3), (4), if the potential $U$ is given in terms of its counterpart $V$, as follows:

$$
\begin{aligned}
U(x)= & V(x)-2 f(x) \frac{\mathrm{d}^{2}}{\mathrm{~d} x^{2}}\{\log [u(x)]\}+ \\
& {\left[\frac{f(x) h^{\prime}(x)}{h(x)}-f^{\prime}(x)\right] \frac{u^{\prime}(x)}{u(x)}-\frac{f^{\prime \prime}(x)}{2}+} \\
& \frac{\left[f^{\prime}(x)\right]^{2}}{4 f(x)}+\frac{3 f(x)\left[h^{\prime}(x)\right]^{2}}{4 h^{2}(x)}-\frac{f(x) h^{\prime \prime}(x)}{h(x)} .
\end{aligned}
$$

Note that (5) remains valid when multiplied by a constant, which can be used for normalization. Now, depending on the choice of the auxiliary solution $u$ in (5), the discrete spectrum of problem (3), (4) can be affected in three possible ways: if $\lambda=E_{0}$ and $u=\psi_{0}$, then $E_{0}$ is removed from the spectrum of (3), (4). The opposite case, creation of a new spectral value $\lambda<E_{0}$, happens if the auxiliary solution $u$ does not fulfill the boundary conditions (4). Finally, the spectra of both problems (1), (2) and (3), (4) are the same, if we pick $\lambda<E_{0}$ and $u$ that fulfills only one of the boundary conditions (2).

Propagator and Green's function. The propagator governs a quantum system's time evolution. For a stationary Schrödinger equation, the propagator $K$ has the defining property

$$
\begin{aligned}
\Psi(x, t)= & \exp (-i E t) \psi(x)= \\
& \int_{(a, b)} K(x, y, t) \psi(y) \mathrm{d} y,
\end{aligned}
$$

as the solution $\Psi$ of the time-dependent Schrödinger equation is related to its stationary counterpart $\psi$ by the exponential factor used for separating time and spatial variable. Suppose problem (1), (2) admits a complete set of eigenfunctions $\left(\psi_{n}\right), n=$ $0,1,2, \ldots, M \in \mathbb{N}_{0}$, where $M$ can stand for infinity, and $\left(\psi_{k}\right), k \in \mathbb{R}$, belonging to the discrete and the continuous part of the spectrum, respectively. Then the propagator $K$ has the representation

$$
\begin{aligned}
K(x, y, t)= & h(y)\left[\sum_{n=0}^{M} \psi_{n}(x) \exp \left(-i E_{n} t\right) \psi_{n}(y)+\right. \\
& \left.\int_{\mathbb{R}} \psi_{k}(x) \exp \left(-i k^{2} t\right) \psi_{k}(y) \mathrm{d} k\right],
\end{aligned}
$$

where $E_{n}$ and $k^{2}$ stand for the spectral values belonging to the discrete and continuous spectrum, respectively. The Green's function $G$ of problem (1), (2) has two equivalent representations [4], both of which we will use here. In order to state the first representation, let $\psi_{0, l}$ and $\psi_{0, r}$ be solutions of equation (1) that fulfill the unilateral boundary conditions $\psi_{0, l}(a)=\psi_{0, r}(b)=0$. The Wronskian $W\left(\psi_{0, l}, \psi_{0, r}\right)$ of these funtions is given by

$$
W\left(\psi_{0, l}, \psi_{0, r}\right)(x)=\frac{c_{0}}{f(x)},
$$

where $c_{0}$ is a constant that depends on the explicit form of $\psi_{0, l}$ and $\psi_{0, r}$. Now we can give the first representation of the Green's function $G_{0}$ for our boundary value problem $(1),(2)$ :

$$
\begin{aligned}
G(x, y)= & -\frac{1}{c_{0}}\left[\psi_{0, l}(y) \psi_{0, r}(x) \theta(x-y)+\right. \\
& \left.\psi_{0, l}(x) \psi_{0, r}(y) \theta(y-x)\right]
\end{aligned}
$$

where $c_{0}$ is the constant from (9) and $\theta$ stands for the Heaviside distribution. The second representation of the Green's function $G$ can be obtained as follows, provided problem (1), (2) admits a complete set of solutions:

$$
\begin{aligned}
G(x, y)= & \sum_{n=0}^{M} \frac{\psi_{n}(x) \psi_{n}(y)}{E_{n}-E}+ \\
& \int_{\mathbb{R}} \frac{\psi_{k}(x) \psi_{k}(y)}{k^{2}-E} \mathrm{~d} k,
\end{aligned}
$$

where the notation is the same as in (8).

\section{Propagators related by generalized SUSY}

In order to obtain a relation between the propagators of the two boundary-value problems (1), (2) and (3), (4), we take the propagator $K_{1}$ of the second problem and express it through quantities related to the first problem. For the sake of simplicity we assume for now that the two boundary-value problems have the 
same discrete spectrum and that both of them admit a complete set of solutions belonging to a discrete and a continuous part of the spectrum. Furthermore, we assume that the solutions of problem (1), (2) are realvalued functions. This is no restriction, as equation (1) involves only real functions.

\subsection{General case}

The construction of our propagator $K_{1}$ is similar to the way it was done in [1]. According to representation (8) we have

$$
\begin{aligned}
K_{1}(x, y, t)= & h(y)\left[\sum_{n=0}^{M} \phi_{n}(x) \exp \left(-i E_{n} t\right) \phi_{n}(y)+\right. \\
& \left.\int_{\mathbb{R}} \phi_{k}(x) \exp \left(-i k^{2} t\right) \phi_{k}(y) \mathrm{d} k\right] .
\end{aligned}
$$

The notation is the same as in (8), only $\phi_{n}$ and $\phi_{k}$ must be replaced by $\psi_{n}$ and $\psi_{k}$, respectively. Now, since the solutions $\phi_{n}, \phi_{k}$ are obtained by means of a SUSY transformation (5) from $\psi_{n}, \psi_{k}$, we can rewrite (12) as follows, taking into account normalization constants $L_{n}$ and $L_{k}$, respectively:

$$
\begin{aligned}
K_{1}(x, y, t)= & h(y) D_{u, x} D_{u, y} . \\
& {\left[\sum_{n=0}^{M} L_{n}^{2} \psi_{n}(x) \exp \left(-i E_{n} t\right) \psi_{n}(y)+\right.} \\
+ & \left.\int_{\mathbb{R}} L_{k}^{2} \psi_{k}(x) \exp \left(-i k^{2} t\right) \psi_{k}(y) \mathrm{d} k\right] .
\end{aligned}
$$

In the next step we apply the defining property (7) to the previously obtained expression:

$$
\begin{aligned}
& K_{1}(x, y, t)=h(y) D_{u, x} D_{u, y} . \\
& {\left[\sum_{n=0}^{M} L_{n}^{2} \int_{(a, b)} K_{0}(x, z, t) \psi_{n}(z) \mathrm{d} z \psi_{n}(y)+\right.} \\
& \left.\int_{\mathbb{R}} L_{k}^{2} \int_{(a, b)} K_{0}(x, z, t) \psi_{k}(z) \mathrm{d} z \psi_{k}(y) \mathrm{d} k\right]
\end{aligned}
$$

We choose the free constants as $L_{n}=1 /\left(E_{n}-\lambda\right)^{\frac{1}{2}}$, $n=1, \ldots, M$, and $L_{k}=1 /\left(k^{2}-\lambda\right)^{\frac{1}{2}}, k \in \mathbb{R}$, where $\lambda$ is the discrete spectral value associated with the auxiliary function $u$. After regrouping terms, these settings render (13) in the form

$$
\begin{aligned}
& K_{1}(x, y, t)=h(y) D_{u, x} D_{u, y}\left\{\int_{(a, b)} K_{0}(x, z, t)\right. \\
& \left.\left[\sum_{n=0}^{M} \frac{\psi_{n}(z) \psi_{n}(y)}{E_{n}-\lambda}+\int_{\mathbb{R}} \frac{\psi_{k}(z) \psi_{k}(y)}{k^{2}-\lambda} \mathrm{d} k\right] \mathrm{d} z\right\}= \\
& h(y) D_{u, x} D_{u, y} \int_{(a, b)} K_{0}(x, z, t) G_{0}(z, y) \mathrm{d} z,
\end{aligned}
$$

where $G_{0}$ is the Green's function of our boundaryvalue problem (1), (2) in its form (11), taken at energy $\lambda$. Relation (15) gives the final connection between the propagators of our two boundary-value problems, provided they admit the same discrete spectrum. In the case that problem (3), (4) admits an additional discrete spectral value $\lambda$ with the corresponding solution $\phi_{-1}$, formula (12) must be modified as follows:

$$
\begin{aligned}
K_{1}(x, y, t)= & h(y)\left[\sum_{n=0}^{M} \phi_{n}(x) \exp \left(-i E_{n} t\right) \phi_{n}(y)+\right. \\
& \phi_{-1}(x) \exp (-i \lambda t) \phi_{-1}(y)+ \\
& \left.\int_{\mathbb{R}} \phi_{k}(x) \exp \left(-i k^{2} t\right) \phi_{k}(y) \mathrm{d} k\right] .
\end{aligned}
$$

From this point, the additional term is maintained until the final relation between the propagators $K_{0}$ and $K_{1}$ results as

$$
\begin{aligned}
& K_{1}(x, y, t)= \\
& h(y)\left\{D_{u, x} D_{u, y}\left[\int_{(a, b)} K_{0}(x, z, t) G_{0}(z, y) \mathrm{d} z\right]+\right. \\
& \left.\phi_{-1}(x) \exp (-i \lambda t) \phi_{-1}(y)\right\},
\end{aligned}
$$

where the Green's function $G_{0}$ is to be taken at energy $\lambda$. Finally, if problem (3), (4) admits one discrete spectral value less than its initial counterpart, formula (12) remains the same except that the sum starts at one instead of at zero. This is maintained until formula (14), where summation now starts at one. Expression (15) then turns into

$$
\begin{aligned}
K_{1}(x, y, t)= & h(y) D_{u, x} D_{u, y} \int_{(a, b)} K_{0}(x, z, t) \\
& \lim _{E \rightarrow E_{0}}\left[G_{0}(z, y)-\frac{\psi_{0}(z) \psi_{0}(y)}{E_{0}-E}\right] \mathrm{d} z,
\end{aligned}
$$

where the Green's function $G_{0}$ is to be taken at energy $E$. In summary, the last three expressions stand for the final relations between the propagators of our two boundary-value problems. Clearly, in the conventional case $h=1$, the above expressions reduce correctly to the known relations [1]. In general, our expressions cannot be simplified anymore, unless more information on the auxiliary solution $u$ is known. We will now study such a case.

\subsection{Special case: ground state as auxiliary solution}

Let us assume that the auxiliary solution $u$ is chosen to be the ground state $\psi_{0}$, associated with the spectral value $E_{0}$, of problem (1), (2). According to our 
brief SUSY review in section 2, this choice implies that the discrete spectrum of problem (3), (4) will not contain the value $E_{0}$ anymore. We will now show that the corresponding relation between the propagators (17), where $u$ is replaced by $\psi_{0}$, can be simplified considerably. While the general procedure of simplification follows a similar way as in the conventional case [1], one must keep track of the nonconstant factor in front of (5). Before we start simplifying (17), we observe that the limit and the operator $D_{\psi_{0}, y}$ in (17) commute, because

$$
\begin{aligned}
& D_{\psi_{0}, y} \lim _{E \rightarrow E_{0}}\left[G_{0}(z, y)-\frac{\psi_{0}(z) \psi_{0}(y)}{E_{0}-E}\right]= \\
& \lim _{E \rightarrow E_{0}}\left[\sum_{n=1}^{M} \frac{\psi_{n}(z) D_{\psi_{0}, y} \psi_{n}(y)}{E_{n}-E}+\right. \\
& \left.\int_{\mathbb{R}} \frac{\psi_{k}(z) D_{\psi_{0}, y} \psi_{k}(y)}{k^{2}-E} \mathrm{~d} k\right] .
\end{aligned}
$$

The first term in the sum vanishes, as $D_{\psi_{0}, y} \psi_{0}(y)=$ 0 , so we obtain

$$
\begin{aligned}
& D_{\psi_{0}, y} \lim _{E \rightarrow E_{0}}\left[G_{0}(z, y)-\frac{\psi_{0}(z) \psi_{0}(y)}{E_{0}-E}\right]= \\
& \lim _{E \rightarrow E_{0}}\left[D_{\psi_{0}, y} G_{0}(z, y)\right] .
\end{aligned}
$$

This property will be useful for rewriting (17). Note that for the sake of simplicity we divide by the factor $h$ :

$$
\begin{aligned}
& \frac{1}{h(y)} K_{1}(x, y, t)= \\
& D_{\psi_{0}, x} \int_{(a, b)} K_{0}(x, z, t) \lim _{E \rightarrow E_{0}}\left[D_{\psi_{0}, y} G_{0}(z, y)\right] \mathrm{d} z= \\
& D_{\psi_{0}, x} \int_{(a, y)} K_{0}(x, z, t) \cdot \\
& \lim _{E \rightarrow E_{0}}\left[-\frac{1}{c_{0}} D_{\psi_{0}, y} \psi_{0, l}(y) \psi_{0, r}(z)\right] \mathrm{d} z+ \\
& D_{\psi_{0}, x} \int_{(y, b)} K_{0}(x, z, t) \cdot \\
& \lim _{E \rightarrow E_{0}}\left[-\frac{1}{c_{0}} \psi_{0, l}(z) D_{\psi_{0}, y} \psi_{0, r}(y)\right] \mathrm{d} z .
\end{aligned}
$$

We will now determine the limits that the integrals contain. To this end, first note that according to (5) the Wronskian $W\left(\psi_{0}, \psi_{0, r}\right)$ is involved in the limits, which we will now find by means of the differential equation that it obeys. We have

$$
\begin{aligned}
& W\left(\psi_{0}, \psi_{0, r}\right)^{\prime}(y)= \\
& \frac{\mathrm{d}}{\mathrm{d} y}\left[\psi_{0}(y) \psi_{0, r}^{\prime}(y)-\psi_{0, r}(y) \psi_{0}^{\prime}(y)\right]= \\
& \psi_{0}(y) \psi_{0, r}^{\prime \prime}(y)-\psi_{0}^{\prime \prime}(y) \psi_{0, r}(y)= \\
& \frac{h(y)}{f(y)} \psi_{0}(y) \psi_{0, r}(y)\left(E_{0}-E\right)-\frac{f^{\prime}(y)}{f(y)} W\left(\psi_{0}, \psi_{0, r}\right)(y) .
\end{aligned}
$$


Rewriting and integrating this equation gives

$$
\begin{aligned}
& \int_{(a, b)} h(z) \psi_{0, l}^{2}(z) \mathrm{d} z= \\
& \int_{(a, b)} \frac{\mathrm{d}}{\mathrm{d} z}\left[f(z) \psi_{0, l}^{\prime}(z)\right] \frac{\partial}{\partial E} \psi_{0, l}(z) \mathrm{d} z- \\
& \int_{(a, b)} \psi_{0, l}(z) \frac{\mathrm{d}}{\mathrm{d} z}\left[f(z) \frac{\partial}{\partial E} \psi_{0, l}^{\prime}(z)\right] \mathrm{d} z
\end{aligned}
$$

The two integrals on the right hand side can each be reformulated using integration by parts.

$$
\begin{aligned}
& \int_{(a, b)} \frac{\mathrm{d}}{\mathrm{d} z}\left[f(z) \psi_{0, l}^{\prime}(z)\right] \frac{\partial}{\partial E} \psi_{0, l}(z) \mathrm{d} z= \\
& \left.\frac{\partial}{\partial E} \psi_{0, l}(z) f(z) \psi_{0, l}(z)\right|_{a} ^{b}- \\
& \int_{(a, b)} f(z) \psi_{0, l}(z) \frac{\partial}{\partial E} \psi_{0, l}^{\prime}(z) \mathrm{d} z \\
& \int_{(a, b)} \psi_{0, l}(z) \frac{\mathrm{d}}{\mathrm{d} z}\left[f(z) \frac{\partial}{\partial E} \psi_{0, l}^{\prime}(z)\right] \mathrm{d} z= \\
& \left.f(z) \psi_{0, l}(z) \frac{\partial}{\partial E} \psi_{0, l}^{\prime}(z)\right|_{a} ^{b}- \\
& \int_{(a, b)} f(z) \psi_{0, l}(z) \frac{\partial}{\partial E} \psi_{0, l}^{\prime}(z) \mathrm{d} z .
\end{aligned}
$$

Observe that both expressions involve the same integral term on their right hand sides. Therefore, if we substitute into their difference above, we obtain

$$
\begin{aligned}
& \int_{(a, b)} h(z) \psi_{0, l}^{2}(z) \mathrm{d} z= \\
& f(b) \psi_{0, l}^{\prime}(b) \frac{\partial}{\partial E} \psi_{0, l}(b)-f(b) \psi_{0, l}(b) \frac{\partial}{\partial E} \psi_{0, l}^{\prime}(b) .
\end{aligned}
$$

Note that in the last step we substituted the limits of integration and made use of the fact that $\psi_{0, l}(a)=0$. Now we are ready to compute the limits in (18). According to (20), the first limit reads

$$
\begin{aligned}
& \lim _{E \rightarrow E_{0}}\left[-\frac{1}{c_{0}} D_{\psi_{0}, y} \psi_{0, l}(y) \psi_{0, r}(z)\right]= \\
& \lim _{E \rightarrow E_{0}}\left[\frac{E_{0}-E}{f(b) \psi_{0, l}(b) \psi_{0, r}^{\prime}(b)} \sqrt{\frac{1}{f(y) h(y)}} \frac{\psi_{0, l}(z)}{\psi_{0}(y)} .\right. \\
& \left.\int_{(y, b)} h(z) \psi_{0}(z) \psi_{0, r}(z) \mathrm{d} z\right]= \\
& \lim _{E \rightarrow E_{0}}\left[\frac{E_{0}-E}{\psi_{0, l}(b)}\right] \cdot \\
& \lim _{E \rightarrow E_{0}}\left[\frac{1}{f(b) \psi_{0, r}^{\prime}(b)} \sqrt{\frac{1}{f(y) h(y)}} \frac{\psi_{0, l}(z)}{\psi_{0}(y)} .\right. \\
& \left.\int_{(y, b)} h(z) \psi_{0}(z) \psi_{0, r}(z) \mathrm{d} z\right]=
\end{aligned}
$$

$$
\begin{aligned}
& -\left.\frac{1}{\frac{\partial}{\partial E} \psi_{0, l}(b)}\right|_{E=E_{0}} . \\
& \lim _{E \rightarrow E_{0}}\left[\frac{1}{f(b) \psi_{0, r}^{\prime}(b)} \sqrt{\frac{1}{f(y) h(y)}} \frac{\psi_{0, l}(z)}{\psi_{0}(y)} .\right. \\
& \left.\int_{(y, b)} h(z) \psi_{0}(z) \psi_{0, r}(z) \mathrm{d} z\right] .
\end{aligned}
$$

In the next step we substitute the first factor using our relation (21), which we need to evaluate at $E=E_{0}$. Since our generalized Schrödinger equation (1) can only have two linearly independent solutions at $E=E_{0}$, the three solutions $\psi_{0, l}, \psi_{0, r}$ and $\psi_{0}$ become linearly dependent there. In particular, they must all fulfill the same boundary conditions (2), which implies for the right hand side of (21) that

$$
\begin{aligned}
& {\left[f(b) \psi_{0, l}^{\prime}(b) \frac{\partial}{\partial E} \psi_{0, l}(b)-\right.} \\
& \left.f(b) \psi_{0, l}(b) \frac{\partial}{\partial E} \psi_{0, l}^{\prime}(b)\right]\left.\right|_{E=E_{0}}= \\
& \left.f(b) \psi_{0, l}^{\prime}(b) \frac{\partial}{\partial E} \psi_{0, l}(b)\right|_{E=E_{0}} .
\end{aligned}
$$

Now, (21) can be solved for $\frac{\partial}{\partial E} \psi_{0, l}(b)$ :

$$
\begin{aligned}
& \left.\frac{\partial}{\partial E} \psi_{0, l}(b)\right|_{E=E_{0}}= \\
& {\left.\left[\frac{1}{f(b) \psi_{0, l}^{\prime}(b)} \int_{(a, b)} h(z) \psi_{0, l}^{2}(z) \mathrm{d} z\right]\right|_{E=E_{0}} .}
\end{aligned}
$$

We use this to replace the first factor of (22) and get

$$
\begin{aligned}
& \lim _{E \rightarrow E_{0}}\left[-\frac{1}{c_{0}} D_{\psi_{0}, y} \psi_{0, l}(y) \psi_{0, r}(z)\right]= \\
& -\lim _{E \rightarrow E_{0}}\left[\frac{\psi_{0, l}^{\prime}(b)}{\psi_{0, r}^{\prime}(b)} \sqrt{\frac{1}{f(y) h(y)}} \frac{\psi_{0, l}(z)}{\psi_{0}(y)} .\right. \\
& \left.\frac{\int_{(y, b)} h(z) \psi_{0}(z) \psi_{0, r}(z) \mathrm{d} z}{\int_{(a, b)} h(z) \psi_{0, l}^{2}(z) \mathrm{d} z}\right] .
\end{aligned}
$$

For taking the limit we recall that at $E=E_{0}$ the functions $\psi_{0, l}, \psi_{0, r}$ and $\psi_{0}$ become linearly dependent. The respective proportionality constants cancel out and we obtain

$$
\begin{aligned}
& \lim _{E \rightarrow E_{0}}\left[-\frac{1}{c_{0}} D_{\psi_{0}, y} \psi_{0, l}(y) \psi_{0, r}(z)\right]= \\
& -\sqrt{\frac{1}{f(y) h(y)}} \frac{\psi_{0}(z)}{\psi_{0}(y)} \frac{\int_{(y, b)} h(z) \psi_{0}^{2}(z) \mathrm{d} z}{\int_{(a, b)} h(z) \psi_{0}^{2}(z) \mathrm{d} z} .
\end{aligned}
$$


The second limit in (18) is found in a similar fashion, yielding

$$
\begin{aligned}
& \lim _{E \rightarrow E_{0}}\left[-\frac{1}{c_{0}} \psi_{0, l}(z) D_{\psi_{0}, y} \psi_{0, r}(y)\right]= \\
& \sqrt{\frac{1}{f(y) h(y)}} \frac{\psi_{0}(z)}{\psi_{0}(y)} \frac{\int_{(a, y)} h(z) \psi_{0}^{2}(z) \mathrm{d} z}{\int_{(a, b)} h(z) \psi_{0}^{2}(z) \mathrm{d} z}
\end{aligned}
$$

note that there is no negative sign in front. Now our two limits can be plugged into the propagator (18):

$$
\begin{aligned}
& \frac{1}{h(y)} K_{1}(x, y, t)=D_{\psi_{0}, x} \int_{(a, y)} K_{0}(x, z, t) \\
& {\left[-\sqrt{\frac{1}{f(y) h(y)}} \frac{\psi_{0}(z)}{\psi_{0}(y)} \frac{\int_{(y, b)} h(w) \psi_{0}^{2}(w) \mathrm{d} w}{\int_{(a, b)} h(w) \psi_{0}^{2}(w) \mathrm{d} w}\right] \mathrm{d} z+} \\
& D_{\psi_{0}, x} \int_{(y, b)} K_{0}(x, z, t) \cdot \\
& {\left[\sqrt{\frac{1}{f(y) h(y)}} \frac{\psi_{0}(z)}{\psi_{0}(y)} \frac{\int_{(a, y)} h(w) \psi_{0}^{2}(w) \mathrm{d} w}{\int_{(a, b)} h(w) \psi_{0}^{2}(w) \mathrm{d} w}\right] \mathrm{d} z .}
\end{aligned}
$$

In order to join the two terms, we rewrite the inner integral over $(y, b)$ as a difference of integrals over $(a, b)$ and $(a, y)$, respectively:

$$
\begin{aligned}
& \frac{1}{h(y)} K_{1}(x, y, t)= \\
& D_{\psi_{0}, x} \int_{(a, y)} K_{0}(x, z, t) . \\
& {\left[-\sqrt{\frac{1}{f(y) h(y)}} \frac{\psi_{0}(z)}{\psi_{0}(y)}\right] \mathrm{d} z+} \\
& D_{\psi_{0}, x} \int_{(a, y)} K_{0}(x, z, t) . \\
& {\left[\sqrt{\frac{1}{f(y) h(y)}} \frac{\psi_{0}(z)}{\psi_{0}(y)} \frac{\int_{(a, y)} h(w) \psi_{0}^{2}(w) \mathrm{d} w}{\int_{(a, b)} h(w) \psi_{0}^{2}(w) \mathrm{d} w}\right] \mathrm{d} z+} \\
& D_{\psi_{0}, x} \int_{(y, b)} K_{0}(x, z, t) \cdot \\
& {\left[\sqrt{\frac{1}{f(y) h(y)}} \frac{\psi_{0}(z)}{\psi_{0}(y)} \frac{\int_{(a, y)} h(w) \psi_{0}^{2}(w) \mathrm{d} w}{\int_{(a, b)} h(w) \psi_{0}^{2}(w) \mathrm{d} w}\right] \mathrm{d} z=} \\
& -\sqrt{\frac{1}{f(y) h(y)}} \frac{1}{\psi_{0}(y)} D_{\psi_{0}, x} . \\
& \int_{(a, y)} K_{0}(x, z, t) \psi_{0}(z) \mathrm{d} z+ \\
& \sqrt{\frac{1}{f(y) h(y)}} \frac{\int_{(a, y)} h(w) \psi_{0}^{2}(w) \mathrm{d} w}{\psi_{0}(y) \int_{(a, b)} h(w) \psi_{0}^{2}(w) \mathrm{d} w} D_{\psi_{0}, x} . \\
& \int_{(a, b)} K_{0}(x, z, t) \psi_{0}(z) \mathrm{d} z .
\end{aligned}
$$

Since according to (5) we have

$$
\begin{aligned}
D_{\psi_{0}, x} \int_{(a, b)} K_{0}(x, z, t) \psi_{0}(z) \mathrm{d} z & = \\
\exp \left(-i E_{0} t\right) D_{\psi_{0}, x} \psi_{0}(x) & =0
\end{aligned}
$$

relation (24) turns after multiplication by $h$ into its final form

$$
\begin{aligned}
K_{1}(x, y, t)= & -\sqrt{\frac{h(y)}{f(y)}} \frac{1}{\psi_{0}(y)} D_{\psi_{0}, x} . \\
& \int_{(a, y)} K_{0}(x, z, t) \psi_{0}(z) \mathrm{d} z .
\end{aligned}
$$

Alternatively, in (23) one can write the inner integral over $(a, y)$ as a difference of integrals over $(a, b)$ and $(y, b)$, respectively. This gives a result slightly different from (25):

$$
\begin{aligned}
K_{1}(x, y, t)= & \sqrt{\frac{h(y)}{f(y)}} \frac{1}{\psi_{0}(y)} D_{\psi_{0}, x} . \\
& \int_{(y, b)} K_{0}(x, z, t) \psi_{0}(z) \mathrm{d} z .
\end{aligned}
$$

It can be seen immediately that for a conventional Schrödinger equation (1) with $f=1$ and $h=1$, our results reduce correctly to the known findings [1].

\section{Concluding remarks}

We have obtained a relation between propagators of generalized Sturm-Liouville problems that are connected by means of SUSY transformations. Our results complement and generalize former findings for the conventional Schrödinger equation [1]. While in the latter reference propagators related by higherorder SUSY transformations are also found to satisfy simple interrelations, the corresponding situation in the generalized case is subject to ongoing research.

\section{References}

[1] Pupasov, A. M., Samsonov, B. F., Günther, U.: Exact propagators for SUSY partners, J. Phys. A 40 (2007), $10557-10589$.

[2] Cooper, F., Khare, A., Sukhatme, U.: Supersymmetry and Quantum Mechanics, Phys. Rep. 251 (1995), 267-388.

[3] Darboux, M. G.: Sur une proposition relative aux équations linéaires, Comptes Rendus Acad. Sci. Paris 94 (1882), 1456-1459.

[4] Duffy, D. G.: Green's functions with applications, Chapman and Hall, New York, 2001.

[5] Fernandez, D. J. C.: Supersymmetric Quantum Mechanics, quant-ph/0910.0192. 
[6] Samsonov, B. F., Sukumar, C. V., Pupasov, A. M.: SUSY transformation of the Green function and a trace formula, J. Phys. A $\mathbf{3 8}$ (2005), 7 557-7565.

[7] Schulze-Halberg, A.: Green's functions and trace formulas for generalized Sturm-Liouville problems related by Darboux transformations, J. Math. Phys. 51 (2010), 053501 (13pp).

[8] Pozdeeva, E., Schulze-Halberg, A.: Trace formula for Green's functions of effective mass Schrödinger equations and N-th order Darboux transformations, Internat. J. Modern Phys. A 23 (2008), 2 635-2 647.
[9] Sukumar, C. V.: Green's functions, sum rules and matrix elements for SUSY partners, J. Phys. A 37 (2004), 10287-10295.

[10] Suzko, A. A., Schulze-Halberg, A.: Darboux transformations and supersymmetry for the generalized Schrödinger equations in $(1+1)$ dimensions, J. Phys. A 42 (2009), 295 203-295 217.

Axel Schulze-Halberg

E-mail: xbataxel@gmail.com

Department of Mathematics and Actuarial Science

Indiana University Northwest

3400 Broadway, Gary, IN 46408, USA 